\title{
Infinite Length Results and Design Implications for Time-Domain Equalizers
}

\author{
Richard K. Martin, Ming Ding, Brian L. Evans, and C. Richard Johnson, Jr. \\ Richard K. Martin and C. Richard Johnson, Jr. \\ Cornell University \\ School of Electrical \& Computer Engineering \\ Ithaca, NY 14853, USA \\ \{frodo, johnson\}@ece.cornell.edu \\ Ming Ding and Brian L. Evans \\ The University of Texas at Austin \\ Dept. of Electrical \& Computer Engineering \\ Austin, TX 78712-1084, USA \\ \{ming, bevans\}@ece.utexas.edu
}

(C) 2003 IEEE. To appear in IEEE Transactions on Signal Processing. Personal use of this material is permitted. However, permission to reprint/republish this material for advertising or promotional purposes or for creating new collective works for resale or redistribution to servers or lists, or to reuse any copyrighted component of this work in other works, must be obtained from the IEEE. Contact:

IEEE Intellectual Property Rights Office

IEEE Service Center

445 Hoes Lane

P.O. Box 1331

Piscataway, NJ 08855-1331, USA.

Telephone: + Intl. 732-562-3966. Fax: + Intl. 732-981-8062. 


\title{
Infinite Length Results and Design Implications for Time-Domain Equalizers
}

\author{
Richard K. Martin*, Student Member, IEEE, Ming Ding, Student Member, IEEE, \\ Brian L. Evans, Senior Member, IEEE, and C. Richard Johnson, Jr., Fellow, IEEE
}

\begin{abstract}
We show that maximum shortening SNR TEQs are often nearly symmetric. Constraining the TEQ to be symmetric causes only a $3 \%$ loss in bit rate (averaged over 8 standard ADSL channels). Symmetric TEQs have greatly reduced design and implementation complexity. We also show that for infinite length TEQs, minimum mean squared error target impulse responses have all zeros on the unit circle, which can lead to poor bit rate performance.
\end{abstract}

Keywords- Multicarrier Modulation, Channel Shortening, Time-domain Equalization, Symmetric Filters.

\section{INTRODUCTION}

$\mathrm{M}$ ULTICARRIER modulation (MCM) techniques such as orthogonal frequency division multiplexing (OFDM) and discrete multi-tone (DMT) have been receiving increasing attention in the literature, and have been deployed in numerous industry standards. MCM is attractive due to the ease with which it can combat channel dispersion.

The system model is shown in Fig. 1. Each $N$ data symbols are passed through an inverse fast Fourier transform (IFFT), which converts the frequency-domain block into a time-domain signal, and an FFT is used at the receiver for demodulation. In order for the subcarriers to be orthogonal, the convolution of the signal and the channel must be a circular convolution. The linear convolution is made to appear circular by adding a cyclic prefix $(\mathrm{CP})$ to each data block, which entails prepending the last $\nu$ samples of each block to the beginning of the block. If the $\mathrm{CP}$ is at least as long as the channel, then the output of each subchannel is equal to the input times a scalar complex gain factor. Each subchannel can then be equalized by a complex scalar, called a frequency-domain equalizer (FEQ). The CP is usually set to a reasonably small value (to limit redundancy), and a time-domain equalizer (TEQ) is employed to shorten the channel to this length. The TEQ is an FIR filter designed such that the delay spread of the effective impulse response is not longer than the CP length [1] - [11].

We use the notation $\mathbf{h}=\left[h_{0}, \cdots, h_{L_{h}}\right], \mathbf{w}=\left[w_{0}, \cdots, w_{L_{w}}\right]$, and $\mathbf{c}=\left[c_{0}, \cdots, c_{L_{c}}\right]$ for the channel, TEQ, and effective channel $(\mathbf{c}=\mathbf{h} \star \mathbf{w})$ impulse responses, respectively; $\tilde{L}_{h}=L_{h}+1$, $\tilde{L}_{w}=L_{w}+1$, and $\tilde{L}_{c}=L_{c}+1$ for the filter lengths; and $\mathbf{A}^{*}$, $\mathbf{A}^{T}, \mathbf{A}^{H}$ for conjugate, transpose, and Hermitian.

This paper analyzes the minimum mean squared error (MMSE) [1] and maximum shortening SNR (MSSNR) [4] TEQ

\footnotetext{
This work was supported in part by NxtWave Communications (now ATI) Langhorne, PA; and The State of Texas Advanced Technology Program under project 003658-0614-2001.

R. K. Martin and C. R. Johnson, Jr., are with the School of Electrical and Computer Engineering, Cornell University, Ithaca, NY, 14853, USA (email: \{frodo,johnson\}@ece.cornell.edu).

M. Ding and B. L. Evans are with the Department of Electrical and Computer Engineering, The University of Texas at Austin, Austin, TX 78712-1084, USA (email: \{ming,bevans\}@ece.utexas.edu).
}

\footnotetext{
*Correspondence: Richard K. Martin, 397 Frank Rhodes Hall, Cornell University, Ithaca, NY, 14853 USA, (607) 254-8819, (607) 255-9072 FAX, frodo@ece.cornell.edu
}

designs. Section II demonstrates existence of symmetry in the TEQ impulse response. Section III analyzes characteristics of the TEQ magnitude response. Section IV discusses the symmetric MSSNR TEQ algorithm, and Section V concludes the paper.

\section{The TEQ IMPUlse RESPONSE}

This section shows that the MSSNR and MMSE designs often lead to TEQs with highly symmetric impulse responses. Sections II-A and II-B review MSSNR and MMSE design methods, respectively. Section II-C examines symmetry in the TEQ and target impulse response (TIR).

\section{A. The MSSNR solution}

The maximum shortening SNR (MSSNR) TEQ design [4] attempts to maximize the ratio of the energy in a window of the effective channel over the energy in the remainder of the effective channel. The MSSNR design was reformulated for numerical stability in [6], and iterative and adaptive implementations have been proposed in [5] and [11]. Following [4], we define

$\mathbf{H}_{w i n}=\left[\begin{array}{cccc}h(\Delta) & h(\Delta-1) & \cdots & h\left(\Delta-\tilde{L}_{w}+1\right) \\ \vdots & & \ddots & \vdots \\ h(\Delta+\nu) & h(\Delta+\nu-1) & \cdots & h\left(\Delta+\nu-\tilde{L}_{w}+1\right)\end{array}\right]$

as the middle $\nu+1$ rows of the (tall) channel convolution matrix $\mathbf{H}$, and $\mathbf{H}_{\text {wall }}$ as the remaining rows of $\mathbf{H}$. Thus, $\mathbf{c}_{\text {win }}=\mathbf{H}_{\text {win }} \mathbf{w}$ yields a length $\nu+1$ window of the effective channel, and $\mathbf{c}_{\text {wall }}=\mathbf{H}_{\text {wall }} \mathbf{w}$ yields the remainder of the effective channel. The MSSNR design problem can be stated as "maximize $\left\|\mathbf{c}_{\text {win }}\right\|$ subject to the constraint $\left\|\mathbf{c}_{\text {wall }}\right\|=1, "[4],[6]$ which reduces to

$$
\max _{\mathbf{w}}(\mathbf{w}^{T} \underbrace{\mathbf{H}_{\text {win }}^{T} \mathbf{H}_{\text {win }}}_{\mathbf{B}} \mathbf{w}) \text { subject to } \mathbf{w}^{T} \underbrace{\mathbf{H}_{\text {wall }}^{T} \mathbf{H}_{\text {wall }}}_{\mathbf{A}} \mathbf{w}=1 .
$$

Solving (2) leads to a TEQ that satisfies the generalized eigenvector problem,

$$
\mathbf{B w}=\lambda \mathbf{A} \mathbf{w}
$$

The solution for $\mathbf{w}$ is the eigenvector corresponding to the largest generalized eigenvalue [12].

\section{B. The MMSE solution}

The minimum mean-squared error (MMSE) design [1], as shown in Fig. 2, creates a virtual target impulse response (TIR) b of length $\nu+1$ such that the MSE (measured between the output of the effective channel and the output of the TIR) is minimized. In the absence of noise, if the input signal is white, then the optimal MMSE and MSSNR solutions are identical [7].

In Fig. 2, the error $e(k)$ is given by

$$
e(k)=\mathbf{c}^{T} \mathbf{x}-\left[\mathbf{0}_{(1 \times \Delta)}, \mathbf{b}^{T}, \mathbf{0}_{\left(1 \times L_{c}-\nu-\Delta\right)}\right] \mathbf{x}+\mathbf{w}^{T} \mathbf{n},
$$




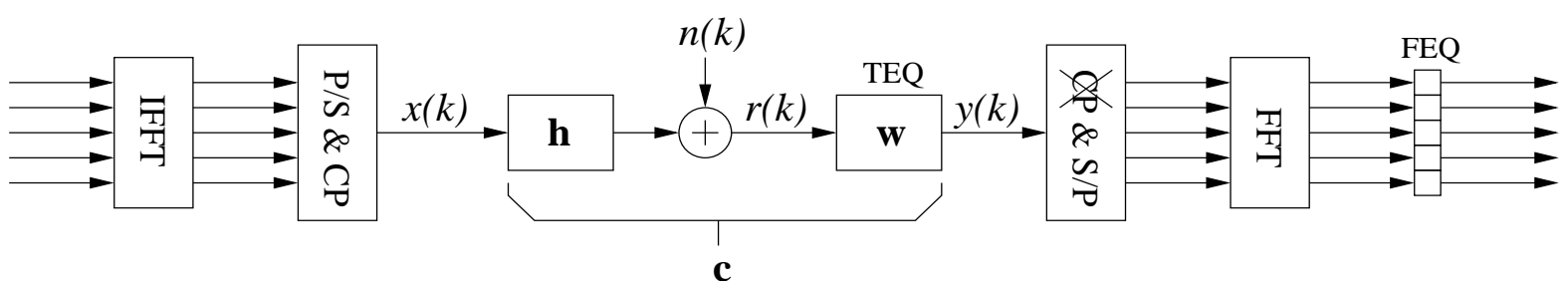

Fig. 1. Traditional multicarrier system model. (I)FFT: (inverse) fast Fourier transform, P/S: parallel to serial, S/P: serial to parallel, CP: add cyclic prefix, and $\mathrm{xCP}$ : remove cyclic prefix.



Fig. 2. MMSE system model: $\mathbf{h}, \mathbf{w}$, and $\mathbf{b}$ are the impulse responses of the channel, TEQ, and target, respectively; and $\Delta$ is a delay. The dashed lines indicate a virtual path, which is used only for analysis.

where $\mathbf{x}$ and $\mathbf{n}$ are vectors of samples of the channel input and the channel noise, respectively. If the input and noise are independent and $x(k)$ is white (i.e. $\left.\mathrm{E}\left[\mathbf{x x}^{T}\right]=\mathbf{I}\right)$, then the MSE is

$$
\begin{aligned}
\mathrm{E}\left[e^{2}(k)\right]= & \left(\mathbf{c}^{T}-\left[\mathbf{0}_{(1 \times \Delta)}, \mathbf{b}^{T}, \mathbf{0}_{\left(1 \times L_{c}-\nu-\Delta\right)}\right]\right) \\
& \cdot\left(\mathbf{c}-\left[\mathbf{0}_{(1 \times \Delta)}, \mathbf{b}^{T}, \mathbf{0}_{\left(1 \times L_{c}-\nu-\Delta\right)}\right]^{T}\right)+\mathbf{w}^{T} \mathbf{R}_{n} \mathbf{w} .
\end{aligned}
$$

For a given $\mathbf{w}$, the choice of $\mathbf{b}$ that minimizes the MSE is a window of the effective channel c. This leads to an MSE of

$$
\begin{aligned}
\mathrm{E}\left[e^{2}(k)\right] & =\mathbf{c}_{\text {wall }}^{T} \mathbf{c}_{\text {wall }}+\mathbf{w}^{T} \mathbf{R}_{n} \mathbf{w} \\
& =\mathbf{w}^{T} \mathbf{H}_{\text {wall }}^{T} \mathbf{H}_{\text {wall }} \mathbf{w}+\mathbf{w}^{T} \mathbf{R}_{n} \mathbf{w} \\
& =\mathbf{w}^{T}\left(\mathbf{A}+\mathbf{R}_{n}\right) \mathbf{w} .
\end{aligned}
$$

The constraint $\|\mathbf{b}\|=1$ is equivalent to $\mathbf{w}^{T} \mathbf{B w}=1$ [7]. Thus, $\mathbf{w}$ is chosen to satisfy

$$
\min _{\mathbf{w}}\left(\mathbf{w}^{T}\left(\mathbf{A}+\mathbf{R}_{n}\right) \mathbf{w}\right) \quad \text { subject to } \mathbf{w}^{T} \mathbf{B} \mathbf{w}=1
$$

The optimal w must satisfy

$$
\mathbf{B w}=\lambda\left(\mathbf{A}+\mathbf{R}_{n}\right) \mathbf{w} .
$$

where $\lambda$ is the largest generalized eigenvalue of the matrix pair $\left(\left(\mathbf{B}, \mathbf{A}+\mathbf{R}_{n}\right)\right)$. The form of (8) allows a theoretical treatment similar to (3).

\section{Symmetry in the TEQ and TIR}

Symmetric centrosymmetric matrices are matrices in the set

$$
V_{N}=\left\{\mathbf{C}: \quad \mathbf{C}^{T}=\mathbf{C}, \quad \mathbf{J C J}=\mathbf{C}\right\},
$$

where $\mathbf{J}$ is the square matrix with ones on the cross diagonal, and zeros elsewhere. $N \times N$ symmetric centrosymmetric matrices have $\lceil N / 2\rceil$ symmetric eigenvectors and $\lfloor N / 2\rfloor$ skewsymmetric eigenvectors [13]. This property can be loosely extended to the generalized eigenvector case.

The channel convolution matrix $\mathbf{H}$ is Toeplitz, so we have $\mathbf{H}^{T} \mathbf{H} \in V_{N}$. Since $\mathbf{A}$ and $\mathbf{B}$ are nearly Toeplitz, this suggests that $\mathbf{A}$ and $\mathbf{B}$ may also be (approximately) in $V_{N}$, since $\mathbf{A}=$ $\mathbf{H}_{\text {wall }}^{T} \mathbf{H}_{\text {wall }}$ and $\mathbf{B}=\mathbf{H}_{\text {win }}^{T} \mathbf{H}_{\text {win }}$. A detailed examination of $\mathbf{A}$ and $\mathbf{B}$ does indeed show that although they are not perfectly centrosymmetric, they are nearly so.

For the MSSNR solution, we must consider the generalized eigenvectors of $(\mathbf{B}, \mathbf{A})$, and for the MMSE solution, we need the generalized eigenvectors of $\left(\mathbf{B},\left(\mathbf{A}+\mathbf{R}_{n}\right)\right)$. However, if $\mathbf{A}$ (or $\left(\mathbf{A}+\mathbf{R}_{n}\right.$ ), for the MMSE case) or $\mathbf{B}$ is invertible, then the generalized eigenvalue problem can be reduced to a traditional eigenvalue problem [12]. When $L_{w}>\nu, \mathbf{H}_{w i n}$ cannot have full column rank, so $\mathbf{B}$ will not be invertible [6]. However, $\mathbf{A}$ and $\left(\mathbf{A}+\mathbf{R}_{n}\right)$ are invertible for all channels longer than the CP.

Recall the generalized eigenvalue problem in (3). Since $\mathbf{A}$ is invertible, w must satisfy

$$
\left(\mathbf{A}^{-1} \mathbf{B}\right) \mathbf{w}=\lambda \mathbf{w}
$$

The inverse of a centrosymmetric matrix is also centrosymmetric [14], and the product of centrosymmetric matrices is centrosymmetric, so $\left(\mathbf{A}^{-1} \mathbf{B}\right)$ is approximately centrosymmetric. Although $\mathbf{A}^{-1}$ and $\mathbf{B}$ are symmetric, $\left(\mathbf{A}^{-1} \mathbf{B}\right)$ may not be, so the full range of results in [13] cannot be immediately applied. One result that still holds is that the eigenvectors are still symmetric or skew-symmetric, although there may not be exactly $\left\lfloor\frac{\tilde{L}_{w}}{2}\right\rfloor$ of symmetric eigenvectors.

Since $\mathbf{A}$ and $\mathbf{B}$ are only approximately in $V_{N}$, the eigenvectors of $\mathbf{A}^{-1} \mathbf{B}$ will only be approximately symmetric or skewsymmetric. (We can replace $\mathbf{A}$ by $\left(\mathbf{A}+\mathbf{R}_{n}\right)$ to obtain similar results for the MMSE case.) Oddly enough, the MSSNR TEQs usually seem to be nearly symmetric rather than nearly skewsymmetric, at least for the carrier serving area (CSA) test loops [10], which are standard test channels for DSL.

To quantify the symmetry, we computed the TEQ coefficients for $3 \leq \tilde{L}_{w} \leq 40$ for CSA test loops 1 through 8 . We decomposed each TEQ $\mathbf{w}$ into $\mathbf{w}_{\text {sym }}$ and $\mathbf{w}_{\text {skew }}$, and then computed $\left\|\mathbf{w}_{\text {skew }}\right\|^{2} /\left\|\mathbf{w}_{\text {sym }}\right\|^{2}$. A plot of this ratio is shown in Fig. 3. The value of $\Delta$ was determined via a global search. The ratios were averaged over the eight CSA loops. The symmetric part was obtained by considering all possible points of symmetry, and choosing the one for which the norm of the symmetric part divided by the norm of the perturbation was maximized. For example, if the TEQ coefficients were $\mathbf{w}=[1,2,4,2.2]$, then $\mathbf{w}_{\text {sym }}=[0,2.1,4,2.1]$ and $\mathbf{w}_{\text {skew }}=[1,-0.1,0,0.1]$. The MSSNR TEQ becomes increasingly symmetric with increasing length. Enforcing symmetry will be discussed in Section IV. The more general case of linear phase TEQs was discussed in [15], through numerical examples.

Now we consider the effects of an infinite length MMSE TEQ. 


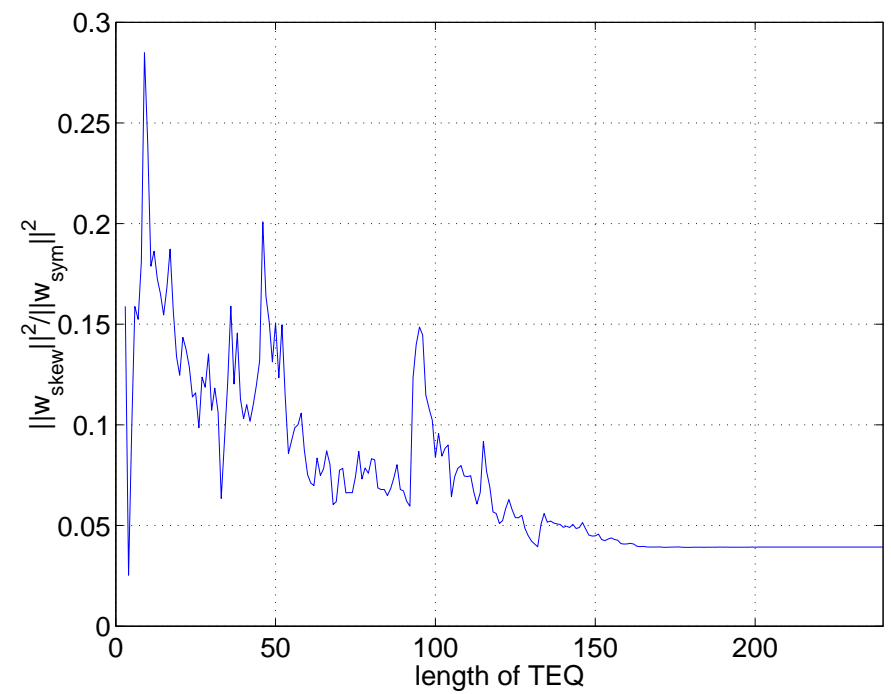

Fig. 3. Energy in the skew-symmetric part of the TEQ over the energy in the symmetric part of the TEQ, for $\nu=32$. The data was delay-optimized and averaged over CSA test loops $1-8$.

Theorem 1: If the input signal is white and the TEQ $\mathbf{w}$ is an infinite length discrete-time filter, then the finite length TIR $\mathbf{b}$ will be symmetric or skew-symmetric.

Remarks: The proof of Theorem 1 is given in the appendix. An outline of the proof of a special case of this theorem was given in [1], which required that the additive channel noise was white, that $\Delta=0$, and that $\mathbf{w}$ was a continuous-time filter. The fact that the TIR in that case was either symmetric or skew-symmetric was pointed out separately in [16].

Thus, for long TEQs, when the symmetry is exploited, the TIR design can be implemented more efficiently with little performance loss. For a finite length TEQ, the TIR should be approximately symmetric. Forcing a symmetric TIR will reduce the computation of $\mathbf{b}$ from an eigendecomposition of a $(\nu+1) \times(\nu+1)$ matrix to an eigendecomposition of a $\lceil(\nu+1) / 2\rceil \times\lceil(\nu+1) / 2\rceil$ matrix. The Matlab code to reproduce the figures in this paper is available in [17].

\section{Characteristics of the magnitude Response}

In this section, we show why the MMSE TEQ has nulls in its magnitude response. Let $\mathbf{R}_{x r}, \mathbf{R}_{x}$, and $\mathbf{R}_{r}$ be the channel input-output cross-correlation, input correlation, and channel output correlation matrices, respectively, and define $\mathbf{R}_{\Delta}=\mathbf{R}_{x}-$ $\mathbf{R}_{x r} \mathbf{R}_{r}^{-1} \mathbf{R}_{r x}$ [1], [3], [8].

Theorem 2: If the input signal is white, the TEQ $\mathbf{w}$ is allowed to be any infinite length discrete-time filter, and the minimum eigenvalue of $\mathbf{R}_{\Delta}$ has multiplicity 1, then the finite length MMSE TIR $\mathbf{b}$ will have all $\nu$ of its zeros on the unit circle.

Proof: The proof of Theorem 1 in the Appendix shows that in the limit $\mathbf{R}_{\Delta}$ becomes a symmetric Toeplitz matrix. Any eigenvector of a symmetric Toeplitz matrix has all of its zeros on the unit circle, if the corresponding eigenvalue has multiplicity 1 [14], [18]. This implies that the TIR (an eigenvector of $\mathbf{R}_{\Delta}$ ) has $\nu$ zeros on the unit circle.

Remarks: 1: A similar result was observed in a footnote in [2], under the three restrictive assumptions noted in the "remarks" after Theorem 1. 2: In the noiseless case with a white input, the MMSE and MSSNR designs produce identical TEQs [7]. The proof of Theorem 1 is still valid in the absence of noise, so long as $\Delta \neq 0$ (examine (A-10) and (A-12) in the appendix). Thus, an

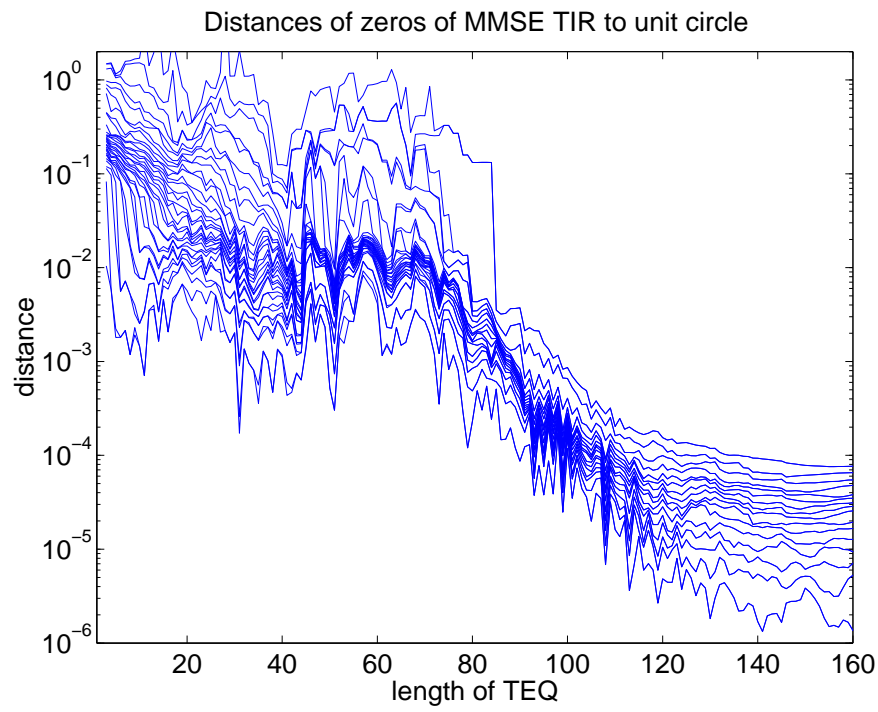

Fig. 4. Distance of the zeros of the MMSE TIR to the unit circle for the case $\nu+1=33$. The values are averaged over CSA loops 1 through 8 . Each curve represents the distance for a single zero.

infinite length MSSNR TEQ will have $\nu$ zeros on the unit circle. 3: If a null lies at one of the subchannel carrier frequencies, then no data can be transmitted in that subchannel [8]. Since bit loading is used, one might expect the loss in bit rate to be mitigated somewhat. However, the loss of $\nu$ subchannels is significant, and it has been observed that the MMSE TEQ often exhibits poor performance for large TEQ lengths [10], and Theorem 2 may account for this.

For a finite length TEQ, $\mathbf{R}_{\Delta}$ is not quite Toeplitz. Thus, the zeros of its eigenvector $\mathbf{b}$ will not be precisely on the unit circle. Fig. 4 plots the average distance of the zeros of the TIR to the unit circle. There are 32 curves, one for each zero. For a length 32 TEQ, the zeros are clustered around a distance of 0.01 from the unit circle, and the asymptotic results agree with Theorem 2 .

\section{Symmetric MSSNR ALGORIThM}

This section presents a practical algorithm for enforcing symmetry in the TEQ. A similar approach was taken in [15], which presented simulations of linear phase equalizers for VDSL channel models. If the TEQ length $\tilde{L}_{w}$ were even, then we could enforce the symmetry by

$$
\mathbf{w}^{T}=\left[\mathbf{v}^{T},(\mathbf{J v})^{T}\right]
$$

and if $\tilde{L}_{w}$ were odd, we could enforce the symmetry by

$$
\mathbf{w}^{T}=\left[\mathbf{v}^{T}, \gamma,(\mathbf{J v})^{T}\right]
$$

where in each case $\mathbf{v}$ has dimensions $\left\lfloor\tilde{L}_{w} / 2\right\rfloor \times 1$. For the evenlength case, the generalized eigenvalue problem of (2) can be simplified via

$$
\begin{gathered}
{\left[\mathbf{v}^{T}, \mathbf{v}^{T} \mathbf{J}\right]\left[\begin{array}{ll}
\mathbf{A}_{11} & \mathbf{A}_{12} \\
\mathbf{A}_{21} & \mathbf{A}_{22}
\end{array}\right]\left[\begin{array}{c}
\mathbf{v} \\
\mathbf{J} \mathbf{v}
\end{array}\right]=} \\
\mathbf{v}^{T} \underbrace{\left[\mathbf{A}_{11}+\mathbf{J} \mathbf{A}_{21}+\mathbf{A}_{12} \mathbf{J}+\mathbf{J} \mathbf{A}_{22} \mathbf{J}\right]}_{\hat{\mathbf{A}}} \mathbf{v}
\end{gathered}
$$


with an analogous definition of $\hat{\mathbf{B}}$ (replace each $\mathbf{A}_{i j}$ with $\mathbf{B}_{i j}$ ). Then the problem becomes

$$
\min _{\mathbf{v}}\left(\mathbf{v}^{T} \hat{\mathbf{A}} \mathbf{v}\right) \quad \text { subject to } \mathbf{v}^{T} \hat{\mathbf{B}} \mathbf{v}=1 .
$$

The solution for $\mathbf{v}$ is the generalized eigenvector of $(\hat{\mathbf{A}}, \hat{\mathbf{B}})$ corresponding to the minimum eigenvalue. This requires a generalized eigendecomposition of symmetric matrices of size $\widetilde{L}_{w} / 2 \times \tilde{L}_{w} / 2$ rather than of size $\tilde{L}_{w} \times \tilde{L}_{w}$, reducing the number of multiply-adds by a factor of 4 . Similar results hold for the odd TEQ length case of (12), though the partioning of $\mathbf{A}$ and $\mathbf{B}$ is slightly different. In either case, we have reduced $\mathbf{A}$ (size $\left.\tilde{L}_{w} \times \tilde{L}_{w}\right)$ to $\hat{\mathbf{A}}\left(\operatorname{size}\left\lceil\tilde{L}_{w} / 2\right\rceil \times\left\lceil\tilde{L}_{w} / 2\right\rceil\right)$.

The matrices $\mathbf{A}$ and $\mathbf{B}$ can be calculated efficiently. It can be shown that $[9]$

$$
\begin{gathered}
\mathbf{A}_{m+1, n+1}=\mathbf{A}_{m, n}-h(\Delta-n) h(\Delta-m) \\
\quad+h(\Delta+\nu-n+1) h(\Delta+\nu-m+1), \\
\mathbf{B}_{m+1, n+1}=\mathbf{B}_{m, n}-h(\Delta+\nu+1-m) h(\Delta+\nu+1-n) \\
+h(\Delta-m) h(\Delta-n) .
\end{gathered}
$$

Fig. 5 shows a computationally efficient method of implementing the proposed method, which has been implemented in the UT Austin DMT TEQ design toolbox [19].

1. Compute $\mathbf{A}$ and $\mathbf{B}$ using (15), then partition them as in (13) to form $\hat{\mathbf{A}}$ and $\hat{\mathbf{B}}$.

2. Solve for $\mathbf{v}$ using a generalized eigendecomposition to find the maximum eigenvalue [20].

(a) Decompose $\hat{\mathbf{B}}=\mathbf{Q R}$, where $\mathbf{Q}$ is orthogonal and $\mathbf{R}$ is upper triangular. Overwrite $\hat{\mathbf{B}}$ with $\mathbf{R}$.

(b) Overwrite $\hat{\mathbf{A}}=\mathbf{Q}^{T} \hat{\mathbf{A}}$.

(c) Hessenberg-Triangular Reduction ([12], p. 380): overwrite $\hat{\mathbf{A}}$ with an upper Hessenberg matrix and $\hat{\mathbf{B}}$ with an upper triangular matrix.

(d) Apply the "QZ" process to matrix pencil $\hat{\mathbf{A}}-\lambda \hat{\mathbf{B}}([12]$ p. 385). It reduces $\hat{\mathbf{A}}$ to upper-quasi triangular form and $\hat{\mathbf{B}}$ to upper triangular form.

(e) Calculate the generalized eigenvalues by dividing each diagonal element of $\hat{\mathbf{A}}$ by the corresponding diagonal element of $\hat{\mathbf{B}}$.

(f) Choose the minimum generalized eigenvalue $\lambda_{\text {min }}$, then iteratively solve $\left(\hat{\mathbf{A}}-\lambda_{\min } \hat{\mathbf{B}}\right) \hat{\mathbf{q}}^{\text {new }}=\hat{\mathbf{B}} \hat{\mathbf{q}}^{\text {old }}$, normalize $\hat{\mathbf{q}}^{\text {new }}=$ $\hat{\mathbf{q}}^{\text {new }} /\left\|\hat{\mathbf{q}}^{\text {new }}\right\|_{2} ;$ using an initial guess of $\hat{\mathbf{q}}^{0}=\mathbf{1}_{\left\lceil\tilde{L}_{w} / 2\right\rceil \times 1}$. Then $\mathbf{v}=\hat{\mathbf{q}}^{\text {final }}$.

3. $\mathbf{w}=\left[\mathbf{v}^{T},(\mathbf{J v})^{T}\right]^{T}$.

Fig. 5. Fast symmetric MSSNR TEQ design method.

Table 1 shows the achievable bit rate using a 32-tap TEQ, for the MSSNR method [4] and the proposed symmetric MSSNR method as in Fig. 5. The channels were the eight standard CSA test loops [10]. The performance loss for the proposed algorithm ranges from $0.1 \%$ (loop 3 ) to $10 \%$ (loop 1), with an average loss of $3 \%$. The results from Table 1 show the same trends as the simulation results in [15] for linear phase equalizers of VDSL channel models.

\section{Conclusions}

For infinite length TEQs, MMSE target impulse responses have all zeros on the unit circle. Hence, finite-length MMSE
TABLE I

AChiEvable BIT RATE (MbPs) USING 32-TAP TEQS AND ADDItive White Gaussian Channel NOISE.

\begin{tabular}{|c|c|c|}
\hline Loop \# & MSSNR & Sym-MSSNR \\
\hline CSA1 & 12.187 & 10.921 \\
CSA2 & 13.016 & 12.493 \\
CSA3 & 11.543 & 11.529 \\
CSA4 & 11.696 & 11.431 \\
CSA5 & 12.120 & 11.800 \\
CSA6 & 10.995 & 10.798 \\
CSA7 & 10.978 & 10.880 \\
CSA8 & 10.294 & 9.956 \\
\hline
\end{tabular}

TEQs eventually yield decreasing bit rate with increasing length. The MSSNR TEQ can be forced to be symmetric. This reduces FIR implementation complexity in half, reduces TEQ training complexity by a factor of 4 , and doubles the length of the TEQ that can be designed using fixed-point arithmetic, with only a small loss in bit rate.

\section{ACKNOWLEDGEMENTS}

The authors would like to thank Mr. Milos Milosevic at The University of Texas at Austin, and Mr. Andrew Klein and Mr. John M. Walsh at Cornell University, for their comments.

\section{APPENDIX}

This appendix proves Theorem 1, by loosely following the less general proof in [1].

Proof: The MMSE solution requires that $\mathbf{R}_{r r} \mathbf{w}=\mathbf{R}_{r x} \mathbf{b}$ [3], which can be simplified to

$$
\mathbf{R}_{n} \mathbf{w}=\mathbf{H}_{w i n}^{T} \mathbf{b}-\mathbf{H}^{T} \mathbf{H} \mathbf{w} .
$$

Allowing $-\infty<i<\infty$, the $i^{\text {th }}$ component becomes

$$
\begin{aligned}
& \sum_{j} R_{n}(i, j) w(j)= \sum_{j=0}^{\nu} h(\Delta+j-i) b(j) \\
&-\sum_{j} \sum_{l} h(l-i) h(l-j) w(j) \\
&= \sum_{j=0}^{\nu} h(\Delta+j-i) b(j) \\
&-\sum_{j} \phi(i-j) w(j),
\end{aligned}
$$

where $\phi(m)=\sum_{l} h(l) h(l+m)$ is the channel covariance function. In convolution notation,

$$
w(i) \star R_{n}(i)=b(i) \star h(\Delta-i)-w(i) \star \phi(i),
$$

where $R_{n}(m)$ is the noise autocorrelation function with $\mathrm{z}$ transform $S_{n}(z)$. Taking z-transforms,

$$
W(z) S_{n}(z)=B(z) z^{-\Delta} H\left(z^{-1}\right)-W(z) \Phi(z) .
$$

Solving for $W(z)$,

$$
W(z)=\frac{z^{-\Delta} B(z) H\left(z^{-1}\right)}{S_{n}(z)+\Phi(z)} .
$$

We will make use of (A-5) shortly. The error sequence is

$$
e(k)=\sum_{l} b(l) x(k-\Delta-l)-\sum_{l} w(l) r(k-l) .
$$


Assuming $x(k)$ is white with unit variance, the error covariance is

$$
\begin{aligned}
E_{m} \triangleq \mathrm{E} & {[e(k) e(k+m)]=\sum_{l} b(l) b(m+l) } \\
& -\sum_{l_{1}, l_{2}} w\left(l_{1}\right) b\left(l_{2}\right) h\left(\Delta-m+l_{2}-l_{1}\right) \\
& -\sum_{l_{1}, l_{2}} w\left(l_{1}\right) b\left(l_{2}\right) h\left(\Delta+m+l_{2}-l_{1}\right) \\
& +\sum_{l_{1}, l_{2}} w\left(l_{1}\right) w\left(l_{2}\right)\left[\phi\left(m+l_{1}-l_{2}\right)+R_{n}\left(m+l_{1}-l_{2}\right)\right] .
\end{aligned}
$$

In convolution notation,

$$
\begin{aligned}
E_{m}= & b(m) \star b(-m)-b(m-\Delta) \star w(\Delta-m) \star h(\Delta-m) \\
& -b(-m-\Delta) \star w(\Delta+m) \star h(\Delta+m) \\
& +w(m) \star w(-m) \star\left[\phi(m)+R_{n}(m)\right] .
\end{aligned}
$$

Taking z-transforms,

$$
\begin{aligned}
E(z)= & B(z) B\left(z^{-1}\right)-z^{-3 \Delta} B(z) W\left(z^{-1}\right) H\left(z^{-1}\right) \\
& -z^{3 \Delta} B\left(z^{-1}\right) W(z) H(z)+W(z) W\left(z^{-1}\right)\left[\Phi(z)+S_{n}(z)\right] .
\end{aligned}
$$

Now insert (A-5) into (A-9). Noting that $\Phi(z)=H(z) H\left(z^{-1}\right)$, and simplifying considerably,

$$
E(z)=B(z) B\left(z^{-1}\right) \underbrace{\left[\frac{S_{n}(z)-\Phi(z)\left(z^{\Delta}-z^{-\Delta}\right)^{2}}{S_{n}(z)+\Phi(z)}\right]}_{G(z)} .
$$

To minimize the MSE, we must minimize $E_{0}$. By setting $z=$ $e^{j \omega}$, taking the inverse Fourier transform, and setting $m=0$, we find that

$$
\begin{aligned}
E_{0} & =\frac{1}{2 \pi} \int_{-\pi}^{\pi} E\left(e^{j \omega}\right) d \omega \\
& =\frac{1}{2 \pi} \int_{-\pi}^{\pi}\left\|b\left(e^{j \omega}\right)\right\|^{2} G\left(e^{j \omega}\right) d \omega
\end{aligned}
$$

where $b\left(e^{j \omega}\right)=\mathbf{b}^{T}\left[1, e^{j \omega}, \ldots, e^{j \omega \nu}\right]^{T}$. This can be rewritten as

$$
\begin{aligned}
E_{0} & =\mathbf{b}^{T} \mathbf{R}_{\Delta} \mathbf{b} \\
{\left[\mathbf{R}_{\Delta}\right]_{m, n} } & =\frac{1}{2 \pi} \int_{-\pi}^{\pi} e^{j \omega(m-n)} G\left(e^{j \omega}\right) d \omega=g(m-n) .
\end{aligned}
$$

Since $S_{n}\left(e^{j \omega}\right)$ and $\Phi\left(e^{j \omega}\right)$ are even functions in $\omega, G\left(e^{j \omega}\right)$ is as well. Thus, $\left[\mathbf{R}_{\Delta}\right]_{m, n}=\left[\mathbf{R}_{\Delta}\right]_{n, m}$, so $\mathbf{R}_{\Delta}$ is a symmetric Toeplitz matrix, and the optimal $\mathbf{b}$ is the eigenvector corresponding to the minimum eigenvalue of $\mathbf{R}_{\Delta}$. By the results in [13], $\mathbf{b}$ will be symmetric or skew-symmetric.

\section{REFERENCES}

[1] D. D. Falconer and F. R. Magee, "Adaptive Channel Memory Truncation for Maximum Likelihood Sequence Estimation," Bell Sys. Tech. Journal, pp. 1541-1562, Nov. 1973.

[2] N. Al-Dhahir, Optimized-Transmitter Reduced-Complexity MMSE-DFE under Finite-Length Constraints, Ph.D. thesis, Stanford University, 1994.

[3] N. Al-Dhahir and J. M. Cioffi, "Efficiently Computed Reduced-Parameter Input-Aided MMSE Equalizers for ML Detection: A Unified Approach," IEEE Trans. on Info. Theory, vol. 42, no. 3, pp. 903-915, May 1996.

[4] P. J. W. Melsa, R. C. Younce, and C. E. Rohrs, "Impulse Response Shortening for Discrete Multitone Transceivers," IEEE Trans. on Comm., vol. 44, pp. 1662-1672, Dec. 1996.

[5] M. Nafie and A. Gatherer, "Time-Domain Equalizer Training for ADSL," in Proc. IEEE Int. Conf. on Comm., Montreal, Canada, June 1997, vol. 2, pp. 1085-1089.

[6] C. Yin and G. Yue, "Optimal Impulse Response Shortening for Discrete Multitone Transceivers," Electronics Letters, vol. 34, pp. 35-36, Jan. 1998.

[7] D. Daly, C. Heneghan, and A. D. Fagan, "A Minimum Mean-Squared Error Interpretation of Residual ISI Channel Shortening for Discrete Multitone Transceivers," in Proc. IEEE Int. Conf. on Acoustics, Speech, and Signal Processing, May 2001, vol. 4, pp. 2065-2068.

[8] B. Farhang-Boroujeny and M. Ding, "Design Methods for Time-Domain Equalizers in DMT Transceivers," IEEE Trans. on Comm., vol. 49, no. 3, pp. 554-562, Mar. 2001.

[9] J. Wu, G. Arslan, and B. L. Evans, "Efficient Matrix Multiplication Methods to Implement a Near-Optimum Channel Shortening Method for Discrete Multitone Transceivers," in Proc. IEEE Asilomar Conf. on Signals, Systems, and Computers, Pacific Grove, CA, Nov. 2000, vol. 1, pp. 152-157.

[10] G. Arslan, B. L. Evans, and S. Kiaei, "Equalization for Discrete Multitone Receivers To Maximize Bit Rate," IEEE Trans. on Signal Processing, vol. 49, no. 12, pp. 3123-3135, Dec. 2001.

[11] R. K. Martin, J. Balakrishnan, W. A. Sethares, and C. R. Johnson, Jr., "A Blind, Adaptive TEQ for Multicarrier Systems," IEEE Signal Processing Letters, vol. 9, no. 11, pp. 341-343, Nov. 2002.

[12] G. H. Golub and C. F. Van Loan, Matrix Computations, third edition, The Johns Hopkins University Press, Baltimore, MD, 1996.

[13] A. Cantoni and P. Butler, "Eigenvalues and Eigenvectors of Symmetric Centrosymmetric Matrices," Linear Algebra and Its Applications, vol. 13, pp. $275-288,1976$.

[14] J. Makhoul, "On the Eigenvectors of Symmetric Toeplitz Matrices," IEEE Trans. on Acoustics, Speech, and Signal Processing, vol. 29, pp. 868-872, Aug. 1981.

[15] C. Ribeiro, V. Silva, and P. S. R. Diniz, "Linear Phase Impulse Response Shortening for xDSL DMT Modems," in IEEE International Telecommunications Symposium, Brazil, Sept. 2002.

[16] A. Cantoni and P. Butler, "Properties of the Eigenvectors of Persymmetric Matrices with Applications to Communication Theory," IEEE Trans. on Comm., vol. 24, pp. 804-809, Aug. 1976.

[17] "Matlab code for papers by R. K. Martin," http://bard.ece.cornell.edu/matlab/martin/index.html

[18] E. Robinson, Statistical Communication and Detection, Griffin, London, 1967

[19] G. Arslan, M. Ding, B. Lu, M. Milosevic, Z. Shen, and B. L. Evans, "MATLAB DMTTEQ Toolbox Version 3.1," The University of Texas at Austin, http://www.ece.utexas.edu/ bevans/projects/adsl/dmtteq/ dmtteq.html.

[20] W. W. Hager, Applied Numerical Linear Algebra, Prentice Hall, Englewood Cliffs, NJ, 1988. 